\section{FATORES DE RISCO PARA ULCERAÇÃO E AMPUTAÇÃO DE EXTREMIDADES INFERIORES EM PORTADORES DE DIABETES MELLITUS}

\author{
Risk factors for lower-extremity ulceration and amputation in \\ patients with diabetes mellitus
}
Factores de riesgo de ulceración y amputación de extremidades inferiores en pacientes con diabetes mellitus

\begin{abstract}
RESUMO
Objetivo: Analisar os fatores de risco para ulceração e amputação de extremidades inferiores em portadores de diabetes mellitus. Métodos: Estudo transversal, quantitativo, realizado em 2013, com 92 indivíduos diabéticos, cadastrados em uma Unidade de Saúde da Família em Maceió, Alagoas, Brasil, através de entrevistas e avaliação clínica dos pés. Investigaram-se as seguintes variáveis: características sociodemográficas (sexo, faixa etária, cor da pele, estado civil, escolaridade e renda) e fatores de risco complementares para o pé diabético (dados clínicos, estilo de vida e prática de autocuidado com os pés). Para identificar a associação entre as variáveis, utilizaram-se os testes Qui-quadrado com correção (Yates), Teste G e Exato de Fisher, sendo considerado o nível de significância de 5\% $(\mathrm{p}<0,05)$. Resultados: A amostra contemplou 92 portadores de diabetes mellitus, sendo $71,7 \%$ (66) do sexo feminino, com faixa etária predominante de 60-79 anos - 48,0\% (44). Observou-se prevalência de $95,6 \%$ (88) com risco para ulceração e 4,4\% (4) com risco para amputação. Com relação ao risco de ulceração, 64,1\% (59) foram classificados em risco 0 (menor risco). Conclusão: Encontrou-se maior prevalência de pacientes em menor risco para ulceração e amputação. Destaca-se o uso inadequado dos calçados como o fator de risco mais comum e o uso do tabaco como fator de risco com associação estatística significativa.
\end{abstract}

Descritores: Diabetes Mellitus; Pé Diabético; Fatores de Risco.

\section{ABSTRACT}

Objective: To analyze the risk factors for lower-extremity ulceration and amputation in patients with diabetes mellitus. Method: Quantitative cross-sectional study conducted in 2013 with 92 individuals with diabetes attending a Family Health Care Center in Maceió, Alagoas, Brazil, through interviews and clinical examination of the feet. The following variables were assessed: sociodemographic characteristics (gender, age group, skin color, marital status, education and income) and additional risk factors for diabetic foot (clinical data, lifestyle and foot self-care practices). Chi-squared test with Yates' correction, G Test and Fisher's Exact test were used to check for association between variables considering a significance level of $5 \%(p<0.05)$. Results: The sample included 92 patients with diabetes mellitus: $71.7 \%$ (66) were women and the predominant age group was $60-79$ years $-48.0 \%$ (44). There was a prevalence of $95.6 \%$ (88) of individuals at risk for ulceration and 4.4\% (4) were at risk for amputation. Regarding the risk of ulceration, $64.1 \%$ (59) were classified as risk 0 (lowest risk). Conclusion: There was a higher prevalence of patients at lower risk for ulceration and amputation. The inappropriate use of shoes stands out as the most common risk factor and tobacco use appears as a risk factor with significant statistical association.

Descriptors: Diabetes Mellitus; Diabetic Foot; Risk Factors.
Artigo Original
1) Universidade Federal de Pernambuco UFPE - Recife (PE) - Brasil

2) Universidade Estadual de Ciências da Saúde de Alagoas - UNCISAL - Maceió (AL) - Brasil
Recebido em: 15/02/2016 Revisado em: 05/04/2016 Aceito em: 11/05/2016 


\section{RESUMEN}

Objetivo: Analizar los factores de riesgo de ulceración y amputación de extremidades inferiores en pacientes con diabetes mellitus. Métodos: Estudio transversal y cuantitativo realizado en 2013 con 92 individuos diabéticos de una Unidad de Salud de la Familia de Maceió, Alagoas, Brasil a través de entrevistas y evaluación clínica de los pies. Se investigaron las siguientes variables: características socio demográficas (el sexo, la franja de edad, el color de la piel, el estado civil, la escolaridad y la renta) y factores de riesgo complementarios para el pie diabético (los datos clínicos, el estilo de vida y la práctica del auto cuidado con los pies). Para la identificación de la asociación entre las variables se utilizó las pruebas Chi-cuadrado con corrección (Yates), la prueba $G$ y el Exacto de Fisher considerando el nivel de significación del 5\% $(p<0,05)$. Resultados: La muestra fue de 92 pacientes con diabetes mellitus, siendo el 71,7\% (66) del sexo femenino y predominio de la franja de edad entre 6079 anos - el 48,0\% (44). Se observó la prevalencia del 95,6\% (88) para el riesgo de ulceración y el 4,4\% (4) para el riesgo de amputación. Respecto al riesgo de ulceración, el 64,1\% (59) se clasificó en riesgo 0 (menor riesgo). Conclusión: Se encontró mayor prevalencia de pacientes con menos riesgo de ulceración y amputación. Se destaca el uso inadecuado de calzados como el factor de riesgo más común y el uso del tabaco como factor de riesgo con asociación estadística significativa.

Descriptores: Diabetes Mellitus; Pie Diabético; Factores de Riesgo.

\section{INTRODUÇÃO}

Com o efetivo envelhecimento da população, as doenças crônicas não transmissíveis tiveram um progressivo aumento na sua incidência. Sendo assim, o diabetes mellitus (DM) tornou-se uma das principais patologias que vêm se destacando pela sua magnitude e gravidade ${ }^{(1,2)}$, constituindo um grave problema de saúde pública no mundo ${ }^{(3)}$.

Atualmente, estima-se que a população mundial portadora de diabetes seja de 387 milhões, podendo alcançar 471 milhões em 2035. No Brasil, em 2014, apontou-se uma prevalência de 11,9 milhões de pessoas com diabetes, na faixa etária de 20 a 79 anos, sendo previsto 19,2 milhões para o ano de $2035^{(4)}$.

DM é uma doença de etiologia heterogênea, caracterizada por hiperglicemia, decorrente da falta de ação de insulina por deficiência de produção desse hormônio, resistência à sua ação, ou de ambas ${ }^{(4,5)}$. Compõe um grupo de doenças metabólicas associadas a complicações, disfunções e insuficiência de diferentes órgãos, podendo atingir principalmente o sistema renal, cardiovascular, neurológico e oftalmológico ${ }^{(6)}$. Essa doença precisa ser avaliada de maneira mais abrangente, devendo-se ampliar a análise à pesquisa, suas complicações e possíveis variáveis de incapacidade, incluindo o pé diabético ${ }^{(1,2)}$.

O termo "pé diabético" é utilizado quando o paciente portador de diabetes apresenta alterações que podem ocorrer de forma isolada ou associada nos pés e membros inferiores, podendo ser alterações neurológicas, infecciosas, ortopédicas e vasculares ${ }^{(7)}$. Trata-se de uma complicação que pode resultar em amputação do membro afetado, cujo surgimento apresenta causas multifatoriais, dentre as quais, a neuropatia sensitivo-motora e autonômica compõe o maior fator de risco ${ }^{(8)}$. Dentre as complicações do diabetes, o pé diabético é uma complicação crônica, considerada a mais grave e de maior impacto socioeconômico ${ }^{(5,9)}$. Representa um custo significativo aos serviços de saúde, relacionado muitas vezes a hospitalização prolongada, custo direto com amputação e cicatrização de úlceras ${ }^{(9)}$.

Como dito anteriormente, a vulnerabilidade ao pé diabético ocorre principalmente por causa da neuropatia periférica, podendo ser dividida em neuropatia sensitiva, causando perda gradual da sensibilidade tátil e dolorosa no pé, neuropatia motora, que gera deformidades ósseas devido à hipotrofia da musculatura interóssea, provocando uma pressão plantar anormal - neuropatia autonômica em que ocorre lesão dos nervos simpáticos, diminuindo o tônus vascular, prejudicando a circulação local e nutrição dos tecidos ${ }^{(2,6,7,9)}$. Além da neuropatia, outro fator desencadeado pelo diabetes é a doença arterial, que se manifesta pela aterosclerose ou isquemia arterial, reduzindo o fluxo sanguíneo necessário para perfusão tecidual adequada. Esse fator, associado a algum trauma, resulta em úlcera, a qual terá seu processo cicatricial prejudicado, podendo levar à gangrena na presença de infecção ${ }^{(6,10)}$.

Fatores associados ao risco para o pé diabético, além da neuropatia, vasculopatia e infecção, incluem o mau controle glicêmico, uso do tabaco, comorbidades (como a hipertensão arterial sistêmica), tempo de diagnóstico do diabetes maior que 10 anos, uso de calçados inadequados, corte das unhas inadequado, bem como úlceras e amputações prévias, que classificam o paciente em um risco mais elevado ${ }^{(7,11)}$.

Para avaliação do paciente diabético, faz-se necessário, inicialmente, a realização de anamnese detalhada e do exame clínico dos pés, em que se pode visualizar a presença de infecções, calos, rachaduras, deformidades ósseas, corte de unhas, amputações prévias, úlceras e diminuição ou ausência dos pulsos nos pés ${ }^{(4,7,8,12)}$, em seguida, a avaliação da sensibilidade cutâneo-plantar, realizada através do monofilamento de Semmes-Weinstein $(10 \mathrm{~g})^{(4,5,7,10)}$. Outro fator a ser analisado é a avaliação dos sinais de doença arterial periférica, feito pela palpação dos pulsos tibial posterior e pedioso, sendo classificados em "presentes" ou "ausentes", sugerindo comprometimento vascular quando não palpáveis ${ }^{(12)}$. 
A melhor maneira custo-benefício de se prevenir as complicações do pé nos portadores de diabetes, como a ulceração e amputação, consiste na identificação dos fatores de risco interrompendo sua progressão ${ }^{(6,7)}$. A atuação dos profissionais da saúde deveria otimizar ações preventivas, como a realização de exames regulares para estratificação do risco, promoção a saúde e hábitos de vida mais saudáveis, educação em saúde com ênfase no autocuidado consciente, identificação da população em risco e intervenções propedêuticas o mais precocemente possível para que ocorra uma diminuição da prevalência de amputações $^{(2,6)}$.

A promoção da saúde é entendida como a capacidade do indivíduo de modificar os determinantes da saúde em benefício da própria qualidade de vida ${ }^{(13)}$. Ainda que seja de competência da equipe de saúde orientar, sensibilizar e motivar as pessoas quanto às transformações para melhoria do autocuidado, os portadores de diabetes precisam aderir e implementar as informações recebidas, gerando um maior nível de independência funcional e mudanças no estilo de vida $^{(14)}$.

Nessa perspectiva, um estudo feito na região Leste de Belo Horizonte pode observar que a participação dos usuários na prática educativa de curto e longo prazo apresenta resultados favoráveis para o controle do diabetes mellitus, bem como suas complicações ${ }^{(15)}$.

Sabe-se que o cuidado holístico ao portador de diabetes é desafiante, principalmente no auxílio da mudança nos hábitos de vida, devido a estes estarem muito ligados às suas condições e interações sociais ${ }^{(7)}$. É imprescindível a atuação de uma equipe multidisciplinar agindo em conjunto para enfrentar os desafios, e assim, desse modo, a equipe de saúde e a comunidade criam um vínculo de corresponsabilidade, facilitando o acompanhamento dos indivíduos para prevenção dos agravos ${ }^{(7)}$.

O presente estudo justifica-se na importância do conhecimento por parte dos profissionais e portadores do DM dos fatores predisponentes para ulceração e amputação de extremidades inferiores. Acredita-se que a identificação desses fatores norteará as intervenções da equipe de saúde, apontando o desenvolvimento de ações preventivas em nível de atenção básica. Portanto, o objetivo do presente estudo foi analisar os fatores de risco para ulceração e amputação de extremidades inferiores em portadores de diabetes mellitus.

\section{MÉTODOS}

Trata-se de estudo transversal, quantitativo, desenvolvido com pacientes diabéticos cadastrados na área 26 de uma Unidade de Saúde da Família, localizada no bairro Trapiche da Barra, no município de Maceió, Alagoas, Brasil, realizado no período de maio a outubro de 2013.
A população diabética cadastrada na área era composta por 118 indivíduos, dos quais 92 participaram do estudo e 26 foram excluídos, de acordo com os critérios de exclusão.

Os critérios de inclusão contemplaram portadores de diabetes mellitus cadastrados na área 26, com idade igual ou superior a 18 anos, que estivessem com sua capacidade de raciocínio e comunicação preservada, sendo excluídos do estudo aqueles que não compareceram às consultas do HIPERDIA nos dias de coleta ou estavam ausentes de sua residência por três visitas em dias e horários alternados, assim como pacientes que mudaram de endereço e/ou faleceram (informação obtida nos registros em prontuários e/ou por familiares).

Os indivíduos eram convidados a participar do estudo de forma particular, esclarecendo-os quanto aos objetivos e procedimentos da pesquisa e, após a aceitação, assinavam o Termo de Consentimento Livre e Esclarecido.

O procedimento de coleta dos dados ocorreu na unidade de saúde nos dias de consulta do HIPERDIA (consulta voltada ao atendimento de hipertensos e diabéticos), semanalmente, no turno da manhã, entrevistando-se em média quatro pacientes novos, sendo o restante captado nas visitas domiciliares em dias e turnos alternados, variando o quantitativo diário, a depender da disponibilidade dos indivíduos. A entrevista ocorreu em locais reservados e confortáveis aos participantes, minimizando os riscos de constrangimento.

As entrevistas e o exame clínico foram realizados por dois pesquisadores devidamente treinados, por meio de um instrumento validado( ${ }^{(6)}$ e adaptado pelos autores do presente estudo. Questionaram-se, inicialmente, dados sociodemográficos, dados clínicos, estilo de vida e prática de autocuidado com os pés; logo após, realizou-se o exame clínico dos pés, a fim de verificar fatores desencadeantes de úlceras e amputações, avaliação de neuropatia utilizando o monofilamento Semmes-Weisntein $(10 \mathrm{~g})$ e avaliação de sinais de doença arterial periférica.

Nos aspectos sociodemográficos, agruparam-se as categorias do estado civil dos pacientes em "com parceiro" para aqueles que referiram ser casados ou estar em uma união estável, e "sem parceiro" para aqueles que relataram ser solteiros, viúvos ou divorciados. Nas práticas de estilo de vida, pesquisou-se o consumo de álcool e/ou tabaco; quanto aos aspectos clínicos, investigou-se a presença de comorbidades autorreferidas que indicassem complicações do diabetes, como hipertensão arterial sistêmica, infarto agudo do miocárdio e acidente vascular encefálico ${ }^{(5)}$.

Avaliou-se a prática de autocuidado com os pés realizada pelos pacientes, questionando-se o hábito de andar descalço. No exame inspecional dos pés, observouse a presença de micoses em unhas ou interdigitais e em 
calçados utilizados no momento da entrevista, sendo estes categorizados em adequados ou inadequados; os calçados considerados adequados eram os que proporcionavam conforto interno, possuíam tamanho ideal e fechamento ajustável (velcro ou cadarço), cobriam os dedos e calcâneo, sendo confeccionados com couro macio ou lona ${ }^{(11)}$.

Para avaliação da neuropatia, aplicou-se o monofilamento de Semmes-Weisntein $(10 \mathrm{~g})$ em seis áreas plantares: primeiro, terceiro e quinto dedos, metatarsos em um ângulo de $90^{\circ}$, com força suficiente para encurvá-lo, e duração de 1 segundo entre as aplicações. O indivíduo, sem visualizar, era questionado quanto à percepção ao toque, sendo duas respostas negativas compatíveis com perda da sensibilidade. Após aplicação em 10 pessoas, colocou-se o monofilamento em descanso por um período de 24 horas, para que não houvesse descalibração, minimizando, assim, erros no diagnóstico ${ }^{(6,10)}$.
Observou-se também a presença de úlceras ou cicatrizes de úlceras (confirmando-se com relato do paciente), amputações prévias e deformidades ósseas - hálux valgo, dedos em garra, dedos em martelo, hipotrofia muscular, proeminências ósseas, dedos sobrepostos e outros que gerem pressão anormal nos pés ${ }^{(7)}$.

$\mathrm{Na}$ análise de sinais de doença arterial periférica, realizou-se palpação dos pulsos tibial posterior e pedioso, sendo classificados como palpáveis ou não palpáveis; a não palpação dos dois pulsos no mesmo membro indicou sinais de comprometimento vascular.

Após a análise dos fatores de risco, os pés dos pacientes foram categorizados em graus de risco para ulceração e amputação $0^{(7)}$. As variáveis utilizadas para estratificação do risco estão descritas no Quadro I.

Para o presente estudo, os riscos foram subdivididos de 0 a 3 como riscos de ulceração, e de $3 \mathrm{~A}$ a $3 \mathrm{E}$ como risco para

Quadro I - Classificação dos pés de portadores de diabetes mellitus em grau de risco para ulceração e amputação.

\begin{tabular}{|c|c|c|c|}
\hline Grau de risco & Sensibilidade & Deformidades/ hiperceratose & Úlceras \\
\hline 0 & Presente & Ausente & Ausente \\
\hline 1 & Ausente & Ausente & Ausente \\
\hline 2 & Ausente & Presente & Ausente \\
\hline 3 & Ausente & Presente ou Ausente & Cicatrizada \\
\hline $3 \mathrm{~A}$ & \multicolumn{3}{|c|}{ Úlcera superficial com ou sem infecção superficial } \\
\hline 3B & \multicolumn{3}{|c|}{ Úlcera profunda, sem infecção e sem atingir osso } \\
\hline $3 \mathrm{C}$ & \multicolumn{3}{|c|}{ Infecção profunda (celulite, abscesso, tendinite, sinovite, osteomielite) } \\
\hline $3 \mathrm{D}$ & \multicolumn{3}{|c|}{ Necrose ou grangrena localizada } \\
\hline $3 \mathrm{E}$ & \multicolumn{3}{|c|}{ Necrose ou gangrena extensa } \\
\hline
\end{tabular}

Fonte: Adaptada da Sociedade Brasileira de Angiologia e Cirurgia Vascular $2001^{(7)}$ e da Classificação de Wagner ${ }^{(7)}$.

amputação. No risco de ulceração, classificaram-se como menor risco os valores de 0 e 1 , e maior risco os valores de 2 e 3 . O risco de amputação subdivide-se em menor risco (3A e 3B) e maior risco (3C, 3D e 3E) $)^{(7)}$.

As informações coletadas ficaram arquivadas no programa Microsoft Excel 2013, sendo posteriormente analisadas pelo programa BioEstat, versão 5.0. Para identificar a associação entre as variáveis, utilizaram-se os testes: Qui-quadrado com correção (Yates), Teste G e Exato de Fisher, com análise bivariada entre as variáveis independentes e a variável dependente (menor e maior risco para ulceração e para amputação), sendo considerado o nível de significância de $5 \%(\mathrm{p}<0,05)$.

O estudo obteve aprovação do Comitê de Ética em Pesquisa da Universidade Estadual de Ciências da Saúde de Alagoas, sob protocolo $\mathrm{n}^{\circ}$. 2034. Todas as informações mantiveram-se confidenciais, sem identificação dos pacientes, ficando sob a responsabilidade do pesquisador.

\section{RESULTADOS}

A população de portadores de diabetes mellitus avaliados totalizou 92 indivíduos, sendo 66 do sexo feminino e 26 do sexo masculino. Os percentuais das características sociodemográficas encontrados na população estão descritos na Tabela I.

Diante do exposto na Tabela I, pôde-se destacar a maior prevalência de indivíduos diabéticos do sexo feminino, faixa etária predominante de 60 a 79 anos, e pacientes com menos de quatro anos de estudo. 
Tabela I - Distribuição dos portadores de diabetes mellitus segundo características sociodemográficas. Maceió, Alagoas, 2013.

\begin{tabular}{|c|c|c|}
\hline \multirow{2}{*}{ Características sociodemográficas } & \multicolumn{2}{|c|}{ Total (92) } \\
\hline & $\mathbf{n}$ & $\%$ \\
\hline \multicolumn{3}{|l|}{ Sexo } \\
\hline Feminino & 66 & 71,7 \\
\hline Masculino & 26 & 28,3 \\
\hline \multicolumn{3}{|l|}{ Estado civil } \\
\hline Com parceiro & 37 & 40,2 \\
\hline Sem parceiro & 55 & 59,8 \\
\hline \multicolumn{3}{|l|}{ Faixa etária (anos) } \\
\hline $18-39$ & 6 & 6,5 \\
\hline $40-59$ & 38 & 41,3 \\
\hline $60-79$ & 44 & 48,0 \\
\hline 80 ou mais & 4 & 4,3 \\
\hline \multicolumn{3}{|l|}{ Cor da pele autorreferida } \\
\hline Branca & 13 & 14,1 \\
\hline Negra & 32 & 34,8 \\
\hline Parda & 47 & 51,1 \\
\hline \multicolumn{3}{|l|}{ Escolaridade } \\
\hline$<4$ anos & 66 & 71,7 \\
\hline 4 a 7 anos & 15 & 16,3 \\
\hline 8 ou mais & 11 & 12,0 \\
\hline \multicolumn{3}{|l|}{ Renda } \\
\hline$<1$ salário mínimo & 9 & 9,8 \\
\hline$>1$ salário mínimo & 20 & 21,7 \\
\hline 1 salário mínimo & 63 & 68,5 \\
\hline
\end{tabular}

Tabela II - Percentagem dos portadores de diabetes mellitus, classificados em graus de risco para ulceração e amputação. Maceió, Alagoas, 2013.

\begin{tabular}{lcc}
\hline Graus de risco & n & Total (92) \\
\cline { 2 - 3 } & & \% \\
\hline Ulceração & 59 & 64,1 \\
Grau 0 & 14 & 15,2 \\
Grau 1 & 3 & 3,3 \\
Grau 2 & 12 & 13,0 \\
Grau 3 & & \\
Amputação & 4 & 4,4 \\
Grau 3A & 0 & 0 \\
Grau 3B & 0 & 0 \\
Grau 3C & 0 & 0 \\
Grau 3D & 0 & 0 \\
Grau 3E & & \\
\hline
\end{tabular}


A Tabela II mostra a distribuição da população estudada, segundo a classificação de risco para ulceração e amputação ${ }^{(7)}$.

Dos 92 indivíduos estudados, observou-se que 95,6\% $(n=88)$ foram classificados em risco para úlceras; destes, $64,1 \%(n=59)$ encontravam-se em risco 0 , compondo a maioria dos indivíduos analisados. Para o risco de amputação, verificou-se a prevalência de $4,4 \%(n=4)$ com risco $3 \mathrm{~A}$, não sendo, portanto, encontrados pacientes nas classificações de 3B a 3E.
Quanto à classificação de risco para ulceração, foram identificados 88 pacientes, dos quais $82,9 \%(n=73)$ se enquadraram em menor risco ( 0 e 1) para úlceras e 17,0\% $(n=15)$, em maior risco (2 e 3$)$.

Identificou-se na população estudada a presença de fatores de risco complementares para complicações nas extremidades inferiores, descritos na Tabela III.

Diante do exposto, ressalta-se a presença de $81,5 \%$ $(n=75)$ de pacientes com HAS associada ao diabetes. A não

Tabela III - Distribuição dos portadores de diabetes mellitus segundo os principais fatores de risco complementares para o pé diabético. Maceió, Alagoas, 2013.

\begin{tabular}{|c|c|c|}
\hline \multirow{2}{*}{ Fatores de risco complementares para o pé diabético } & \multicolumn{2}{|c|}{ Total (92) } \\
\hline & $\mathbf{n}$ & $\%$ \\
\hline \multicolumn{3}{|l|}{ Tempo de diagnóstico de DM } \\
\hline Até 10 anos & 64 & 69,6 \\
\hline Maior que 10 anos & 28 & 30,4 \\
\hline \multicolumn{3}{|l|}{ Comorbidades } \\
\hline \multicolumn{3}{|l|}{ HAS } \\
\hline Sim & 75 & 81,5 \\
\hline Não & 17 & 18,5 \\
\hline \multicolumn{3}{|l|}{ AVE } \\
\hline Sim & 8 & 8,7 \\
\hline Não & 84 & 91,3 \\
\hline \multicolumn{3}{|l|}{ IAM } \\
\hline Sim & 5 & 5,4 \\
\hline Não & 87 & 94,6 \\
\hline \multicolumn{3}{|l|}{ Consumo de álcool } \\
\hline Sim & 14 & 15,2 \\
\hline Não & 78 & 84,8 \\
\hline \multicolumn{3}{|l|}{ Consumo de tabaco } \\
\hline Sim & 12 & 13,0 \\
\hline Não & 46 & 50,0 \\
\hline Ex-fumante & 34 & 37,0 \\
\hline \multicolumn{3}{|l|}{ Calçado utilizado no momento } \\
\hline Adequado & 12 & 13,0 \\
\hline Inadequado & 80 & 87,0 \\
\hline \multicolumn{3}{|l|}{ Caminha descalço } \\
\hline Sim & 36 & 39,1 \\
\hline Não & 56 & 60,9 \\
\hline \multicolumn{3}{|l|}{ Presença de micose em unhas/dedos } \\
\hline $\operatorname{Sim}$ & 19 & 20,6 \\
\hline Não & 73 & 79,4 \\
\hline \multicolumn{3}{|l|}{ Alteração do pulso } \\
\hline Sim & 18 & 19,6 \\
\hline Não & 74 & 80,4 \\
\hline
\end{tabular}

DM: Diabetes Mellitus; HAS: Hipertensão Arterial Sistêmica; IAM: Infarto Agudo do Miocárdio; AVE: Acidente Vascular Encefálico. 
Tabela IV - Distribuição de portadores de diabetes mellitus segundo as principais variáveis estudadas e a classificação de risco para ulceração. Maceió, Alagoas, 2013.

\begin{tabular}{|c|c|c|c|c|c|c|c|}
\hline \multirow{3}{*}{ Características } & \multicolumn{4}{|c|}{ Classificação de risco para ulceração } & & & \multirow{3}{*}{ Valor de $p$} \\
\hline & \multicolumn{2}{|c|}{$\begin{array}{c}\text { Maior risco } \\
\text { (15) }\end{array}$} & \multicolumn{2}{|c|}{$\begin{array}{l}\text { Menor risco } \\
(73)\end{array}$} & \multicolumn{2}{|c|}{ Total (88) } & \\
\hline & $\mathbf{n}$ & $\%$ & $\mathbf{n}$ & $\%$ & $\mathbf{n}$ & $\%$ & \\
\hline \multicolumn{8}{|c|}{ Tempo de Diagnóstico do DM } \\
\hline Até 10 anos & 8 & 53,3 & 53 & 72,6 & 61 & 69,3 & \multirow{2}{*}{$0,2170^{\#}$} \\
\hline Maior que 10 anos & 7 & 46,7 & 20 & 27,4 & 27 & 30,7 & \\
\hline \multicolumn{8}{|l|}{ Comorbidades } \\
\hline \multicolumn{8}{|l|}{ HAS } \\
\hline Sim & 12 & 80,0 & 59 & 80,8 & 71 & 80,7 & \multirow[b]{2}{*}{$0,9923^{\#}$} \\
\hline Não & 3 & 20,0 & 14 & 19,2 & 17 & 19,3 & \\
\hline \multicolumn{8}{|l|}{ IAM } \\
\hline Sim & 0 & 0,0 & 5 & 6,8 & 5 & 5,7 & \multirow[b]{2}{*}{$0,5883^{\#}$} \\
\hline Não & 15 & 100,0 & 68 & 93,1 & 83 & 94,3 & \\
\hline \multicolumn{8}{|l|}{ AVE } \\
\hline $\operatorname{Sim}$ & 2 & 13,3 & 5 & 6,8 & 7 & 7,9 & \multirow[b]{2}{*}{$0,5965^{\#}$} \\
\hline Não & 13 & 86,7 & 68 & 93,1 & 81 & 92,0 & \\
\hline \multicolumn{8}{|l|}{ Consumo do álcool } \\
\hline Sim & 3 & 20,0 & 10 & 13,7 & 13 & 14,8 & \multirow{2}{*}{$0,6878^{\#}$} \\
\hline Não & 12 & 80,0 & 63 & 86,3 & 75 & 85,2 & \\
\hline \multicolumn{8}{|l|}{ Consumo de tabaco } \\
\hline Sim & 2 & 13,3 & 10 & 13,7 & 12 & 13,6 & \multirow{3}{*}{$0,0106^{*}$} \\
\hline Não & 12 & 80,0 & 32 & 43,8 & 44 & 50,0 & \\
\hline Ex-fumante & 1 & 6,7 & 31 & 42,5 & 32 & 36,4 & \\
\hline \multicolumn{8}{|c|}{ Calçado utilizado no momento } \\
\hline $\begin{array}{l}\text { Adequado } \\
\text { Inadequado }\end{array}$ & $\begin{array}{c}2 \\
13\end{array}$ & $\begin{array}{l}13,3 \\
86,7\end{array}$ & $\begin{array}{c}9 \\
64\end{array}$ & $\begin{array}{l}12,3 \\
87,7\end{array}$ & $\begin{array}{l}11 \\
77\end{array}$ & $\begin{array}{l}12,5 \\
87,5\end{array}$ & $0,9814^{\#}$ \\
\hline \multicolumn{8}{|l|}{ Caminha descalço } \\
\hline Sim & 4 & 26,7 & 32 & 43,8 & 36 & 40,9 & \multirow{2}{*}{$0,3454^{\circ}$} \\
\hline Não & 11 & 73,3 & 41 & 56,2 & 52 & 59,1 & \\
\hline \multicolumn{8}{|c|}{ Presença de micose em unhas/dedos } \\
\hline Sim & 6 & 40,0 & 12 & 16,4 & 18 & 20,4 & \multirow{2}{*}{$0,0721^{\#}$} \\
\hline Não & 9 & 60,0 & 61 & 83,6 & 70 & 79,6 & \\
\hline \multicolumn{8}{|l|}{ Alteração do pulso } \\
\hline Sim & 5 & 33,3 & 12 & 16,4 & 17 & 19,3 & \multirow{2}{*}{$0,1556^{\#}$} \\
\hline Não & 10 & 66,7 & 61 & 83,6 & 71 & 80,7 & \\
\hline
\end{tabular}

\# Teste Exato de Fisher; *Teste G; oTeste Qui-quadrado. DM: Diabetes Mellitus; HAS: Hipertensão Arterial Sistêmica; IAM: Infarto Agudo do Miocárdio; AVE: Acidente Vascular Encefálico.

palpação do pulso tibial posterior e pedioso no mesmo membro teve um percentual de $19,6 \%(\mathrm{n}=18)$ nos pacientes estudados. Os calçados inadequados em uso no momento da entrevista alcançaram um índice de 87,0\% $(\mathrm{n}=80)$ na população investigada.

Realizou-se uma análise bivariada da associação entre a frequência de maior e menor risco para ulceração em pés de portadores de DM com características sociodemográficas, dados clínicos, estilo de vida, práticas de autocuidado, alterações dermatológicas e vasculares, como descrito na Tabela IV. Ressalta-se que não foram encontrados pacientes com maior risco para amputação, dessa forma, não foi possível realizar uma análise bivariada para risco de amputação, sendo feita apenas para ulceração.
De acordo com a Tabela IV, encontrou-se apenas associação significativa em relação ao uso do tabaco e ao risco de úlceras $(\mathrm{p}=0,010)$.

A análise bivariada com os dados socioeconômicos não trouxe associação significativa.

\section{DISCUSSÃO}

No que diz respeito às características sociodemográficas, pôde-se perceber uma maior predominância de portadores de diabetes do sexo feminino $(71,7 \%)$ no presente estudo, fato encontrado também por outros estudos ${ }^{(6,16-18)}$. Esse episódio pode estar relacionado ao fato de as mulheres terem uma maior expectativa de vida em relação aos homens, 
conseguindo alcançar a vida adulta e envelhecimento ${ }^{(19)}$, bem como estarem mais preocupadas com sua saúde, procurando o serviço de saúde de atenção primária, visando medidas preventivas, ao contrário da população masculina, que procura mais o serviço terciário e quando há $\mathrm{o}$ acometimento de complicações patológicas ${ }^{(20,21)}$.

A Sociedade Brasileira de Diabetes ${ }^{(10)}$ refere a faixa etária de 60 a 69 anos como a de maior prevalência para o diabetes, condizendo com os achados do presente estudo; resultados semelhantes foram encontrados por outros autores ${ }^{(18,22,23)}$.

A baixa escolaridade dos indivíduos é um fator de risco complementar para a não adesão de práticas no autocuidado e ainda um grande desafio para as equipes de saúde ${ }^{(24)}$. Além da alta prevalência de diabéticos com baixo grau escolar identificada no atual estudo, outros autores ${ }^{(25)}$ trazem resultados semelhantes, com prevalência de $40,5 \%$ de pacientes diabéticos analfabetos, e um estudo internacional ${ }^{(23)}$ aponta prevalência de $60,0 \%$ de diabéticos com até 5 anos de estudo, sendo, destes, 25,0\% analfabetos.

No presente estudo, nos aspectos econômicos, notouse uma maior proporção da população que se mantém com apenas um salário mínimo $(68,5 \%)$. É imprescindível ressaltar a importância do nível de conhecimento dos portadores de diabetes e suas condições econômicas, para aumentar seu grau de potencialidade com práticas preventivas de complicações ${ }^{(24)}$; para tal, a equipe de saúde tem de corroborar com medidas educativas relativas ao tratamento, tanto ao paciente como aos familiares.

A atuação familiar no contexto de pacientes portadores de doenças crônicas é primordial, inserindo cuidados para auxílio nas mudanças alimentares, realização de atividade física, monitorização da glicemia, adesão ao tratamento e prática de cuidado com os pés, principalmente aos pacientes impossibilitados de se cuidarem sozinhos ${ }^{(26,27)}$.

$\mathrm{Na}$ atual pesquisa, a cor da pele autorreferida que prevaleceu foi a parda $(51,1 \%)$, seguida da cor negra $(34,8 \%)$. A Sociedade Brasileira de Diabetes ${ }^{(10)}$ apregoa importantes diferenças da prevalência de diabetes de acordo com países e grupos étnicos. Nessa esfera, vale elucidar que, além da susceptibilidade genética, há a influência dos fatores externos, como estilo de vida e padrão alimentar.

Para estratificação em graus de risco de ulceração e amputação, a alta prevalência de pacientes com baixo ou nenhum risco para ulceração $(82,9 \%)$ foi um fator positivo identificado no presente estudo, e uma pesquisa ${ }^{(17)}$ com objetivo comum trouxe uma prevalência de 56,0\% de diabéticos com risco 0 para ulceração e $16,0 \%$ de indivíduos classificados em grau 3. Em Ribeirão Preto, estudo semelhante ${ }^{(24)}$ também evidenciou uma alta prevalência de diabéticos em risco 0 para úlceras $(79,8 \%) \mathrm{e}$ $14,0 \%$ apresentando risco 3 . Vale elucidar, entretanto, que, apesar de os pacientes investigados no estudo em questão serem classificados em graus de risco baixo para ulceração e amputação, eles apresentaram práticas inadequadas de autocuidado com os pés e estilo de vida, como também tinham um baixo nível escolar, contribuindo, dessa maneira, para o surgimento de maiores complicações crônicas.

O tempo de diagnóstico da doença é um fator importante para o aumento do risco de complicações nos pés, sendo o tempo $>10$ anos um fator de risco complementar para o surgimento do pé diabético ${ }^{(6)}$. Os dados obtidos na presente pesquisa identificaram que a maioria dos pacientes tinha um tempo de diagnóstico menor que 10 anos, porém, não foi encontrada associação significativa entre o tempo de diagnóstico e prevalência de risco para ulceração.

A hipertensão arterial sistêmica tem alta prevalência nos portadores de diabetes, sendo um fator de risco para doenças cardiovasculares e comprometimento microvascular, propiciando o surgimento de retinopatia e nefropatia ${ }^{(28)}$. Esse fato foi de encontro com os achados da presente pesquisa, em que $81,5 \%$ dos diabéticos tinham como comorbidade a hipertensão arterial, apresentando-se em risco para o surgimento de complicações importantes do diabetes, achado encontrado por outro estudo ${ }^{(26)}$.

Um importante fator de risco para o pé diabético é o tabaco, pois sua presença aumenta a ocorrência de alterações macrovasculares que podem aumentar o surgimento de úlceras, como também compromete seu prognóstico quando estas já estão presentes, propiciando o aparecimento de complicações como infecção, gangrena e até amputação ${ }^{(26,29,30)}$. Destaca-se no presente estudo a associação significativa do uso do tabaco com o risco para ulceração, embora, dos 88 indivíduos em risco para úlceras, $50,0 \%$ da população referiu não fazer uso do fumo e $36,4 \%$ referiram ser ex-fumantes em um período maior que seis meses. Destarte, a associação pode ter ocorrido indicando que o hábito de não fumar é um fator preventivo ao aparecimento de úlceras.

A presença de micose nos espaços interdigitais e ungueais serve como porta de entrada para infecções, sendo estas um dos principais fatores desencadeantes de amputação em membros inferiores ${ }^{(1)}$. Pesquisas evidenciam prevalência significativa de micoses interdigitais ${ }^{(6,31)}$. Esse tipo de alteração dermatológica representa um elevado fator de risco para complicações no pé diabético, necessitando de avaliação e monitoramento contínuo por parte dos profissionais de saúde, atuação com medidas preventivas, tratamento e acompanhamento de lesões, visando sua melhoria e reintegração do tecido epitelial ${ }^{(25)}$.

Na realização de investigação da alteração dos pulsos através da palpação, 19,6\% dos pacientes apresentaram alteração dos pulsos tibial posterior e pedioso no mesmo membro, sendo estes não palpáveis, sugerindo 
comprometimento vascular. As alterações vasculares são consideradas como um dos principais determinantes de aparecimento de úlceras e dificuldades no processo de cicatrização, evoluindo para amputação ${ }^{(5-7)}$.

Outro fator relacionado ao surgimento de úlceras e consequente amputação nas extremidades de portadores de diabetes é a presença de hábitos inadequados de autocuidado com os pés, como o uso de calçados impróprios, aumentando o risco externo de lesões nos pés e surgimento de calos ${ }^{(7,32)}$. No presente estudo, foi observada uma frequência de $87,0 \%$ de indivíduos utilizando calçados inadequados no momento da entrevista.

Os achados pelos autores, associados aos encontrados em outros estudos aqui descritos, demonstraram a necessidade de avaliação das equipes de saúde, para propor medidas de intervenções mais eficazes às necessidades e vulnerabilidades dos indivíduos diabéticos. Ressaltase, então, a importância da continuidade da avaliação de fatores de risco para a ulceração e amputação e da melhoria na qualidade da assistência prestada para a população diabética, com a ampliação de intervenções e programas que foquem principalmente o incentivo de práticas de promoção da saúde e estilos de vida mais saudáveis ${ }^{(7,32,33)}$.

Este estudo apresentou algumas limitações que devem ser consideradas, como o universo estudado em que se permitiu considerar resultados relacionados a um único grupo de pacientes diabéticos, porém, ressalta-se que os resultados encontrados corroboraram com os de estudos semelhantes, intensificando os dados obtidos. Outro fator limitante foi a perda de indivíduos na amostra pelo não comparecimento nas consultas, dificultando o encontro dos mesmos ao domicilio. Destarte, faz-se necessário o contínuo planejamento de ações que contribuam para prevenção de complicações e melhoria da qualidade de vida desses indivíduos.

\section{CONCLUSÃO}

Encontrou-se maior prevalência de pacientes em menor risco para ulceração e amputação de extremidades inferiores, detectando o uso do tabaco como um fator de risco estatisticamente significativo e o uso de calçados inadequados como fator de risco complementar mais prevalente.

\section{AGRADECIMENTO}

À Fundação de Amparo à Pesquisa do Estado de Alagoas - FAPEAL, à Universidade Estadual de Ciências da Saúde de Alagoas e à Unidade de Saúde Virgem dos Pobres III.

\section{REFERÊNCIAS}

1. Santos ICRV, Carvalho EF, Souza WV, Albuquerque EC. Factors associated with diabetic foot amputations. J Vasc Bras. 2015;14(1):37-45.

2. Santos ICRV, Sobreira CMM, Nunes ENS, Morais MCA. Prevalência e fatores associados a amputações por pé diabético. Ciênc Saúde Coletiva. 2013;18(10):3007-14.

3. Magalhães AT, Silva BAK, Ribeiro JA, Aguiar JF Bisneto, Pereira LPI, Machado NV, et al. Avaliação do risco de desenvolver diabets mellitus tipo $2 \mathrm{em}$ população universitária. Rev Bras Promoç Saúde. 2015;28(1):5-15.

4. Sociedade Brasileira de Diabetes. Diretrizes da Sociedade Brasileira de Diabetes 2015-2016. São Paulo: AC Farmacêutica; 2016.

5. Ministério da Saúde (BR), Secretaria de Atenção à Saúde, Departamento de Atenção Básica. Estratégias para o cuidado da pessoa com doença crônica: diabetes mellitus. Brasília: Ministério da Saúde; 2013.

6. Bortoletto MSS, Haddad MCL, Karino ME. Pé diabético, uma avaliação sistematizada. Arq Ciênc Saúde Unipar. 2009;13(1):37-43.

7. Caifa JS, Castro AA, Fidelis C, Santos VP, Silva ES, Sitrângulo CJ Jr. Atenção integral ao portador de pé diabético. J Vasc Bras. 2011;10(4 Supl 2):1-32.

8. Escola de Saúde Pública do Estado de Minas Gerais. Curso de Atualização Profissional em Manejo Clínico do Pé Diabético. Minas Gerais: Secretaria de Estado de Saúde de Minas Gerais; 2010.

9. Oliveira AF, Marchi ACB, Leguisamo CP, Baldo GV, Wawginiak TA. Estimativa do custo de tratar o pé diabético, como prevenir e economizar recursos. Ciênc Saúde Coletiva. 2014;19(6):1663-71.

10. Sociedade Brasileira de Diabetes. Diretrizes da Sociedade Brasileira de Diabetes 2013-2014. São Paulo: AC Farmacêutica; 2014.

11. Ochoa-Vigo K, Pace EA. Pé diabético: estratégias para prevenção. Acta Paul Enferm. 2005;18(1):100-9.

12. Armstrong DG, Boulton AJM, Albert SF, Frykberg RG, Hellman R, Kirkman MS, et al. Comprehensive Foot Examination and Risk Assessment. Diabetes Care. 2008;31(8):1679-85.

13. Szwarcwald CL, Souza PR Júnior, Damacena GN, Almeida WD, Malta DC, Stopa SR, et al. Recommendations and practice of healthy behaviors among patients with diagnosis and diabetes in Brazil: National Health Survey (PNS), 2013. Rev. Bras. Epidemiol. 2015;18(Supl 2):132-45. 
14. Cubas MR, Santos OM, Retzlaff EMA. Pé diabético: orientações e conhecimentos sobre cuidados preventivos. Fisioter Mov. 2013;26(3):647-55.

15. Maia MA, Reis IA, Torres HC. Relationship between the users' contact time in educational programs on diabetes mellitus and self-care skills and knowledge. Rev Esc Enferm USP. 2016;50(1):59-64.

16. Dourado MA, Santos ICRV. Adesão aos cuidados de prevenção do pé diabético. Revista Estima. 2015;13(4):1-6.

17. Audi EG, Moreira RC, Moreira ACMG, Pinheiro EFC, Mantovani MF, Araújo AG. Avaliação dos pés e classificação do risco para pé diabético: contribuição da enfermagem. Cogitare Enferm. 2011;16(2):240-6.

18. Rodrigues DF, Brito GEG, Sousa NM, Rufino TMS, Carvalho TD. Prevalência de Fatores de Risco e Complicações do Diabetes Mellitus Tipo 2 em Usuários de uma Unidade de Saúde da Família. Rev Bras Ciênc Saúde. 2011;15(3):277-86.

19. Instituto Brasileiro de Geografia e Estatística. Síntese de Indicadores Sociais. Rio de Janeiro: IBGE; 2013.

20. Cotta RMM, Batista KCS, Reis RS, Souza A, Dias G, Castro FAF, Alfenas RCG, et al. Perfil sociossanitário e estilo de vida de hipertensos e/ou diabéticos, usuários do Programa de Saúde da Família no município de Teixeiras, MG. Ciênc Saúde Coletiva. 2009;14(4): 1251-60.

21. Batista MCR, Priore SE, Rosado LEFPL, Tinôco ALA, Franceschini SCC. Avaliação dos resultados da atenção multiprofissional sobre o controle glicêmico, perfil lipídico e estado nutricional de diabéticos atendidos em nível primário. Rev Nutr. 2005;18(2):219-28.

22. Santos ICRV, Nunes ENS, Melo CA, Farias DG. Amputações por pé diabético e fatores sociais: implicações para cuidados preventivos de enfermagem. Rev Rene. 2011;12(4):684-91.

23. Hernández FM, Reza CG, Martinez VG, Guadarrama FC. Cuidado de los pies en usuarios que viven con diabetes en el estado de México: bases para la sistematización de la asístencia de enfermeira. Enferm Foco (Brasília). 2011;2(1):23-7.

24. Pace AE, Foss MC, Ochoa-Vigo K, Hayashida M. Fatores de risco para complicações em extremidades inferiores de pessoas com diabetes mellitus. Rev Bras Enferm. 2002;55(5):514-21.

25. Araújo MFM, Gonçalves TC, Damasceno MMC, Caetano JA. Aderência de diabéticos ao tratamento medicamentoso com hipoglicemiantes orais. E s c Anna Nery Rev Enferm. 2010;14(2):361-7.
26. Karino ME, Pace AE. Risco para complicações em pés de trabalhadores portadores de diabetes mellitus. Ciênc Cuid Saúde. 2012;11(Supl):183-90.

27. Pace AE, Nunes PD, Ochoa-Vigo K. O conhecimento dos familiares acerca da problemática do portador de diabetes mellitus. Rev Latinoam Enferm. 2003;11(3):312-9.

28. Moreschi C, Rempel C, Carreno I, Silva DS, Pombo CNF, Cano MRL. Prevalência e perfil das pessoas com diabetes cadastradas no Sistema de Informação da Atenção Básica (SIAB). Rev Bras Promoç Saúde. 2015;28(2):184-90.

29. Alonso-Fernández M, Mediavilla-Bravo JJ, LópezSimarro F, Comas-Samper JM, Carramiñana-Barrera F, Mancera-Romero J, et al. Evaluation of diabetic foot screening in Primary Care. Endocrinol Nutr. 2014;61(6):311-7.

30. Chan ACRV, Lima PF, Chaves JBC, Raymundo CS. Incidência de amputação em membros inferiores associada a diabetes mellitus. Saúde Colet (Barueri Impr). 2009;6(33):222-6.

31. Queiróz IWO, Gonçalves O, Faria CCC, Dias JML. Análise dos fatores desencadeantes do pé diabético em uma Unidade de Atenção Primária à Saúde. Perquirere. 2012;9(1):70-80.

32. Oliveira PS, Bezerra EP, Andrade LL, Soares MJGO, Costa MML. Fatores de risco para complicações decorrentes do Diabetes Mellitus. Rev Enferm UFPE. 2013;7(8):5265-73.

33. Stopa SR, César CL, Segri NJ, Goldbaum M, Guimarães VM, Alves MC, et al. Self-reported diabetes in older people comparison of prevalences and control measures. Rev Saúde Pública. 2014;48(4):554-62.

\section{Endereço do primeiro autor:}

Thaysa Alves Tavares

Universidade Federal de Pernambuco - UFPE

Hospital das Clínicas

Travessa Professor Moraes Rego, 1235

Bairro: Cidade Universitária

CEP 50670-901- Recife - PE - Brasil

E-mail: alves.thaysa@yahoo.com.br

\section{Endereço para correspondência:}

Luana Jeniffer Souza Farias da Costa

Universidade Estadual de Ciências da Saúde de Alagoas UNCISAL

Rua Jorge de Lima, 113

Bairro: Trapiche da Barra

CEP: 57010-300- Maceió - AL - Brasil

E-mail: lua-souza@hotmail.com 